\title{
Detection of copeptin in peripheral blood of patients with aneurysmal subarachnoid hemorrhage
}

\author{
Xiang-Dong Zhu* ${ }^{*}$, Jing-Sen Chen, Feng Zhou, Qi-Chang Liu, Gao Chen and Jian-Min Zhang
}

\begin{abstract}
Introduction: Copeptin has been proposed as a prognostic marker in acute illness. This study investigated the ability of copeptin to predict the disease outcome and cerebrovasospasm in the patients with aneurysmal subarachnoid hemorrhage.

Methods: In this retrospective study, 303 consecutive patients were included. Upon admission, plasma copeptin levels were measured by enzyme-linked immunosorbent assay. The end points were mortality after 1 year, inhospital mortality, cerebrovasospasm and poor functional outcome (Glasgow Outcome Scale score of 1-3) after 1 year.

Results: Upon admission, plasma copeptin level in patients was statistically significantly higher than that in healthy controls. A multivariate analysis showed that plasma copeptin level was an independent predictor of poor functional outcome and mortality after 1 year, in-hospital mortality and cerebrovasospasm. A receiver operating characteristic curve showed that plasma copeptin level on admission predicted poor functional outcome and mortality after 1 year, in-hospital mortality and cerebrovasospasm of patients statistically significantly. The area under curve of the copeptin concentration was similar to those of World Federation of Neurological Surgeons (WFNS) score and modified Fisher score for the prediction of poor functional outcome and mortality after 1 year, and in-hospital mortality, but not for the prediction of cerebrovasospasm. In a combined logistic-regression model, copeptin improved the area under curve of WFNS score and modified Fisher score for the prediction of poor functional outcome after 1 year, but not for the prediction of mortality after 1 year, in-hospital mortality, and cerebrovasospasm.
\end{abstract}

Conclusions: Copeptin level is a useful, complementary tool to predict functional outcome and mortality after aneurysmal subarachnoid hemorrhage.

\section{Introduction}

Copeptin, the C-terminal part of the arginine vasopressin precursor peptide, is associated with the severity and outcome of critical illness, and therefore, has been proposed as a prognostic marker in acute illness [1-11]. Recently, it has been reported that plasma copeptin levels were also elevated in the patients with traumatic brain injury $[11,12]$ and intracerebral hemorrhage $[10,13]$ and ischemic stroke [8,9]; in these groups of patients, high copeptin levels were highly predictive for

\footnotetext{
* Correspondence: hzzhuxiangdong@163.com

† Contributed equally

Department of Neurosurgery, The Second Affiliated Hospital, School of

Medicine, Zhejiang University, 88 Jiefang Road, Hangzhou 310000, PR China
}

poor outcome. However, No published information exists to date about the association of copeptin with disease outcome and cerebrovasospasm after aneurysmal subarachnoid hemorrhage (SAH). The present study aimed to investigate the ability of copeptin to predict the disease outcome and cerebrovasospasm in the patients with aneurysmal SAH.

\section{Materials and methods \\ Study population}

Between July 2008 and March 2010, all patients with aneurysmal SAH confirmed by computerized tomography $(\mathrm{CT})$ angiography with or without digital subtraction angiography who were admitted to Department of 
Neurosurgery, Second Affiliated Hospital, School of Medicine, Zhejiang University were evaluated in the study. Inclusion criteria were clinical history of SAH within the last 24 hrs before admission and the treatment by surgery or coiling within the $48 \mathrm{hrs}$ after admission. Exclusion criteria were less than 18 years of age, existing previous head trauma, neurological diseases including ischemic or hemorrhagic stroke, use of antiplatelet or anticoagulant medication, and presence of other prior systemic diseases including uremia, liver cirrhosis, malignancy, chronic heart or lung disease, diabetes mellitus and hypertension.

A control group consisted of 150 healthy sex and agematched subjects with normal results on brain magnetic resonance imaging and without vascular risk factors.

Written informed consent to participate in the study was obtained from the subjects or their relatives. This protocol was approved by the Ethics Committee of The Second Affiliated Hospital, School of Medicine, Zhejiang University before implementation.

\section{Clinical and radiological assessment}

On arrival to the emergency department, a detailed history of vascular risk factors, concomitant medication, Glasgow Coma Scale (GCS) score, body temperature, heart rate, respiratory rate and blood pressure were taken. At admission, clinical severity was assessed using the World Federation of Neurological Surgeons (WFNS) score [14]. The initial CT was classified according to the modified Fisher score [15]. All CT scans were performed according to the neuroradiology department protocol. Investigators who read them were blinded to clinical information.

\section{Patient management}

The type of treatment (surgery or coiling) was decided according to both location and size of the aneurysm by the neurosurgeon and the neuroradiologist. All patients received intravenous Nimodipine at a dose of $2 \mathrm{mg} / \mathrm{h}$ from admission until at least day 14, except during periods of uncontrolled increased intracranial pressure during which intravenous Nimodipine was discontinued. Seizures were systematically prevented by Sodium Valproate (200 $\mathrm{mg} \times 3$, per os). After surgery or coiling, those patients who had delayed ischemic neurological deficit or cerebrovasospasm were managed with triple $\mathrm{H}^{\prime}$ therapy (hypertension with a mean arterial pressure goal greater than $100 \mathrm{~mm} \mathrm{Hg}$, hypervolemia and hemodilution with a goal hematocrit of 30) through 12 days after hemorrhage. An external ventricular drain was inserted in case of hydrocephalus on CT and in patients with a high WFNS grade (WFNS score of 3-5). Increased intracranial pressure was treated by cerebrospinal fluid drainage, mechanical ventilation, reinforcement of sedation, and, rarely, moderate hypothermia. CT was performed whenever clinical deterioration occurred to search for secondary complications such as hydrocephalus or ischemia.

Clinical onset of cerebral vasospasm was defined as the acute onset of a focal neurologic deficit or a change in the GCS score of 2 or more points. All suspected cases of cerebral vasospasms were confirmed by CT angiography and were then taken to the interventional radiology suite for cerebral angiography. Each vasospasm episode was treated with intra-arterial administration of Nimodipine as recently described. This therapy was repeated if necessary. Balloon Angioplasty was used as a second-line therapy when Nimodipine was judged insufficient. Computed tomography ischemia was referred to as delayed ischemia attributed to vasospasm.

\section{Determination of copeptin in plasma}

The informed consents were obtained from study population or family members in all cases before the blood were collected. In the control group, venous blood was drawn at study entry. In the SAH patients, venous blood was drawn on admission. The blood samples were immediately placed into sterile EDTA test tubes and centrifuged at $1500 \mathrm{~g}$ for 20 minutes at $4^{\circ} \mathrm{C}$ to collect plasma. Plasma was stored at $-70^{\circ} \mathrm{C}$ until assayed. The concentration of copeptin in plasma was analyzed by enzyme-linked immunosorbent assay (ELISA) using commercial kits (Cusabio biotech co. ltd, Wuhan, Hubei Province, China) in accordance with the manufactures' instructions. Intra-assay and inter-assay coefficients of variation were $4.2 \%$ and $6.8 \%$. The blood samples were run in duplicate. Researchers running ELISAs were blinded to all patient details.

\section{End point}

Participants were followed up until death or completion of one year after SAH. Their primary outcome was death (at 1 year or in-hospital) and their secondary outcomes were vasospasm and functional outcome at 1 year. The functional outcome was defined by Glasgow outcome scale (GOS) score. GOS was defined as follows: 1 = death; 2 = persistent vegetative state; $3=$ severe disability; $4=$ moderate disability; and $5=$ good recovery [16]. GOS Scores were dichotomized in good and poor functional outcomes (GOS of 4-5 vs. GOS of 1-3). For follow-up, we used structure telephone interviews performed by 1 doctor, blinded to clinical information and copeptin levels.

\section{Statistical analysis}

Statistical analysis was performed with SPSS 10.0 (SPSS Inc., Chicago, IL, USA) and MedCalc 9.6.4.0. (MedCalc Software, Mariakerke, Belgium). The normality of data 
distribution was assessed by the Kolmogorovor-Smirnov test or Shapiro-Wilk test. All values are expressed as mean \pm standard deviation or counts (percentage) unless otherwise specified. Comparisons were made by using (1) chi-square test or Fisher exact test for categorical data, (2) unpaired Student $t$ test for continuous normally distributed variables, and (3) the Mann-Whitney U-test for continuous non-normally distributed variables. The relations of copeptin to the poor functional outcome (GOS 1-3), death and cerebrovasospasm were assessed in a binary logistic-regression model. For multivariate analysis, we included the significantly different outcome predictors as assessed in univariate analysis. A receiver operating characteristic curve was configured to establish the cutoff point of plasma copeptin with the optimal sensitivity and specificity for predicting the poor functional outcome (GOS 1-3), death and cerebrovasospasm. In a combined logistic-regression model, we estimated the additive benefit of copeptin to other predictors (WFNS grade and Fisher grade). A $P$ value of less than 0.05 was considered statistically significant.

\section{Results}

\section{Study population characteristics}

During the recruitment period, 347 patients were admitted with an initial diagnosis of aneurysmal SAH, $312(89.9 \%)$ patients fulfilled the inclusion criteria, and adequate data on admission and follow-up were available for 303 individuals (87.3\%) who were finally included in the analysis (Figure 1). Table 1 summarized the demographic, clinical, laboratory and radiological data of the patients.

\section{The change in plasma copeptin level on admission in patients with SAH}

After SAH, plasma copeptin level on admission in patients was statistically significantly higher than that in healthy controls $(21.2 \pm 9.0 \mathrm{pmol} / \mathrm{L}$ vs. $6.4 \pm 1.8 \mathrm{pmol} /$ L; $P<0.001)$ (Figure 2).

\section{One-year mortality prediction}

Forty-two patients (13.9\%) died from SAH in one year. Higher plasma copeptin level was associated with oneyear mortality, as well as other variables shown in the Table 2. When the above variables found to be significant in the univariate analysis were introduced into the logistic model, A multivariate analysis selected WFNS score, modified Fisher score and plasma copeptin level as the independent predictors for one-year mortality of patients (Table 3).

A receiver operating characteristic curve showed that plasma copeptin level on admission predicted one-year mortality of patients statistically significantly (Figure $3 \mathrm{~A})$. The predictive value of the copeptin concentration was similar to those of WFNS score and modified Fisher score (Table 4). In a combined logistic-regression model, copeptin did not statistically significantly improved the area under curve of WFNS score $(P=0.125)$ and modified Fisher score $(P=0.164)$.

\section{In-hospital mortality prediction}

Thirty-two patients (10.6\%) died from SAH in the hospital. Higher plasma copeptin level was associated with inhospital mortality, as well as other variables shown in the Table 5. When the above variables found to be significant in the univariate analysis were introduced into the logistic model, a multivariate analysis selected WFNS score, modified Fisher score and plasma copeptin level as the independent predictors for in-hospital mortality of patients (Table 6).

A receiver operating characteristic curve showed that plasma copeptin level on admission predicted in-hospital mortality of patients statistically significantly (Figure 3B). The predictive value of the copeptin concentration was similar to those of WFNS score and modified Fisher score (Table 7). In a combined logistic-regression model, copeptin did not statistically significantly improved the area under curve of WFNS score $(P=$ $0.148)$ and modified Fisher score $(P=0.135)$.

\section{Cerebrovasospasm prediction}

One hundred and thirty-one (43.2\%) suffered from cerebrovasospasm in the hospital. Higher plasma copeptin level was associated with cerebrovasospasm, as well as other variables shown in the Table 8 . When the above variables found to be significant in the univariate analysis were introduced into the logistic model, a multivariate analysis selected WFNS score, modified Fisher score and plasma copeptin level as the independent predictors for cerebrovasospasm of patients (Table 9).

A receiver operating characteristic curve showed that plasma copeptin level on admission predicted cerebrovasospasm of patients statistically significantly (Figure 3C). The predictive value of the copeptin concentration was lower than those of WFNS score and modified Fisher score (Table 10). In a combined logistic-regression model, copeptin did not statistically significantly improved the area under curve of WFNS score $(P=$ $0.206)$ and modified Fisher score $(P=0.288)$.

\section{Poor neurologic function prediction}

Ninety patients $(29.7 \%)$ suffered from poor neurologic outcome (GOS 1-3) in one year. Higher plasma copeptin level was associated with one-year poor neurologic outcome, as well as other variables shown in the Table 11 . When the above variables found to be significant in the univariate analysis were introduced into the logistic model, a multivariate analysis selected WFNS score, 


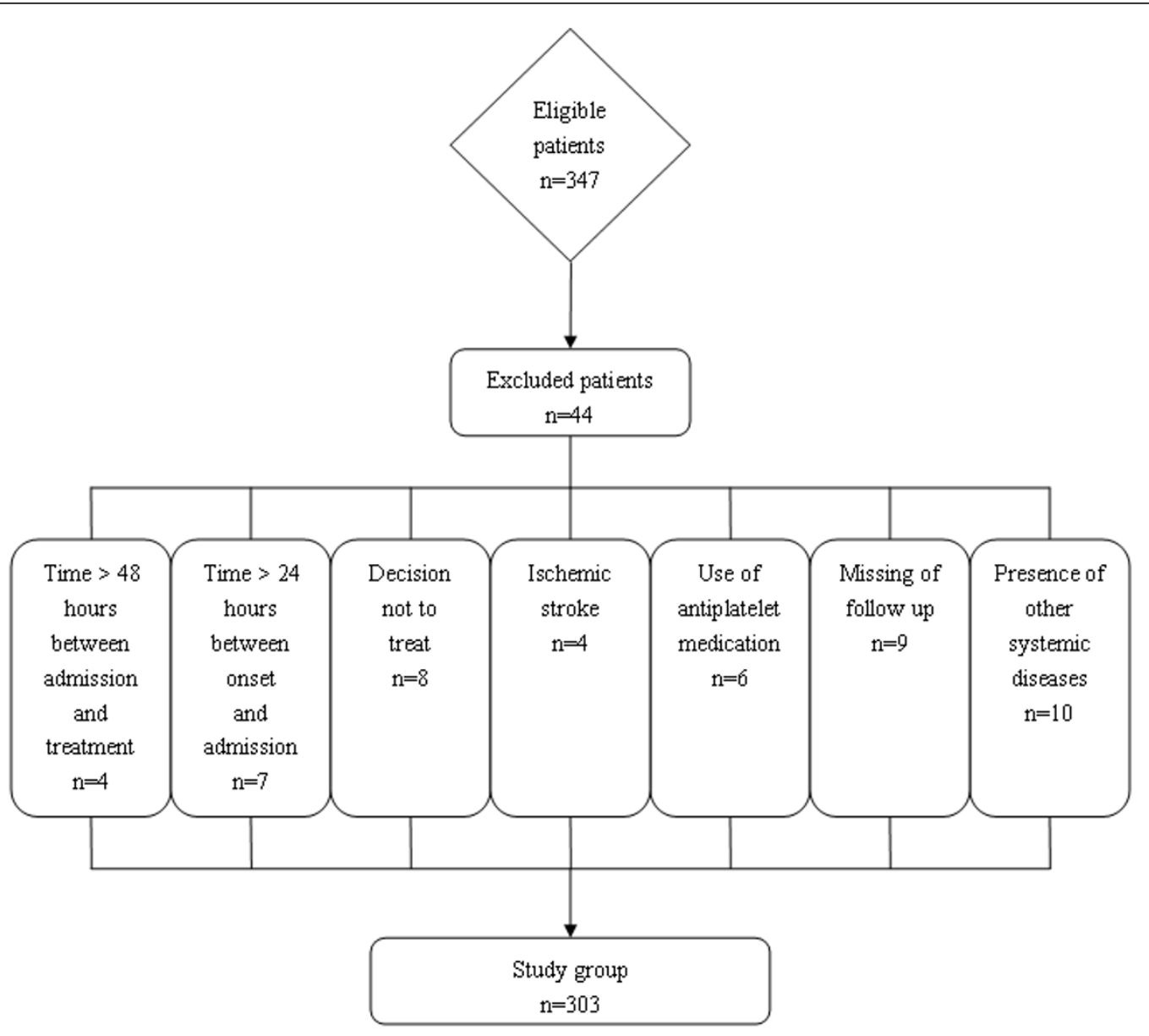

Figure 1 Graph documenting patients' entry into the study from screening.

modified Fisher score and plasma copeptin level as the independent predictors for one-year poor neurologic outcome of patients (Table 12).

A receiver operating characteristic curve showed that plasma copeptin level on admission predicted one-year poor neurologic outcome of patients statistically significantly (Figure 3D). The predictive value of the copeptin concentration was similar to those of WFNS score and modified Fisher score (Table 13). In a combined logistic-regression model, copeptin statistically significantly improved the area under curve of WFNS score $(P=$ $0.018)$ and modified Fisher score $(P=0.029)$.

\section{Discussion}

In this retrospective study, we aimed to investigate the plasma copeptin levels in the SAH patients, and therefore, presence of other prior systemic diseases including uremia, liver cirrhosis, malignancy, chronic heart or lung disease, diabetes mellitus and hypertension, that may be associated with plasma copeptin levels $[1-7,17]$, may become the confounding variables, and finally, were excluded. Furthermore, we demonstrated that plasma copeptin levels on admission in the patients were significantly higher than those in healthy controls; and in patients who had poor functional outcome or died in a year or die in hospital, the copeptin levels on admission were significantly higher compared with levels in survivors or patients with good functional outcome. In multivariate logistic regression models of predictors of death and poor functional outcome that included other confounding variables previously reported [18-20], the copeptin levels on admission were an independent predictor. Need to mention, of two patients with basilar tip aneurysms in this study, one refused to treat and the other had missing of follow up. And therefore, they could be included in this study. In addition, in this study, plasma copeptin level for these patients (turning point: $23.8 \mathrm{pmol} / \mathrm{L}$ ) was similar to that of intracerebral hemorrhage (turning point: $18 \mathrm{pmol} / \mathrm{L}$ ) [9] or ischemic stroke (turning point: $16.3 \mathrm{pmol} / \mathrm{L}$ ) [10] in previous reports. Grade III is typically associated with worse poorer outcome. This result was verified by receiver 
Table 1 The characteristics for 303 patients

\begin{tabular}{|c|c|}
\hline Characteristics & \\
\hline Sex (male/female) & $131 / 172$ \\
\hline Age (y) & $\begin{array}{c}43.9 \pm \\
12.4\end{array}$ \\
\hline $\begin{array}{l}\text { World Federation of Neurological Surgeons score on } \\
\text { admission }\end{array}$ & $2.3 \pm 1.2$ \\
\hline Modified Fisher score on admission & $2.7 \pm 1.0$ \\
\hline \multicolumn{2}{|l|}{ Aneurysmal location } \\
\hline Posterior communication artery & $83(27.4 \%)$ \\
\hline Internal carotid artery & $43(14.2 \%)$ \\
\hline Anterior communication artery & $66(21.8 \%)$ \\
\hline Middle cerebral artery & $45(14.9 \%)$ \\
\hline Anterior cerebral artery & $35(11.6 \%)$ \\
\hline Posterior cerebral artery & $23(7.6 \%)$ \\
\hline Vertebral artery & $8(2.6 \%)$ \\
\hline Surgery & $\begin{array}{c}186 \\
(61.4 \%)\end{array}$ \\
\hline Aneurysmal size (mm) & $7.2 \pm 4.9$ \\
\hline Rebleeding & $16(5.3 \%)$ \\
\hline Acute hydrocephalus & $90(29.7 \%)$ \\
\hline Intracerebral hemorrhage & $39(12.9 \%)$ \\
\hline Intraventricular hemorrhage & $72(23.8 \%)$ \\
\hline External ventricular drain & $\begin{array}{c}109 \\
(36.0 \%)\end{array}$ \\
\hline Angiographic vasospasm & $\begin{array}{c}131 \\
(43.2 \%)\end{array}$ \\
\hline Computed tomography ischemia & $50(16.5 \%)$ \\
\hline Admission time (hr) & $4.7 \pm 3.6$ \\
\hline Plasma-sampling time (hr) & $6.7 \pm 4.4$ \\
\hline Seizure & $44(14.5 \%)$ \\
\hline Plasma C-reactive protein level (mg/L) & $7.1 \pm 2.7$ \\
\hline plasma D-dimer level (mg/L) & $2.1 \pm 0.9$ \\
\hline Plasma copeptin level (pmol/L) & $21.2 \pm 9.0$ \\
\hline
\end{tabular}

operating characteristic curve analysis with high area under curve. Recently, various risk factors for poor outcome after SAH have been identified, and include age, WFNS grade, aneurysmal size, vasospasm, clot thickness and so on [17]. In this study, aneurysmal size, vasospasm, computed tomography ischemia and so on were highly associated with poor outcome in univariate analysis, but they were not in multivariate analysis. However, WFNS grade, modified Fisher grade and plasma copeptin level were identified as independent predictors for poor outcome. Generally, in few reports, biochemical markers were assessed. In this study, some biochemical markers were included. However, if larger sample size are obtained, these factors such as vasospasm and computed tomography ischemia, which are probably equally important, will be included multivariate model. And therefore, it is possible that different variables included in these studies and study designs led to these differences. In addition, in previous exploratory analysis, aneurysm coiling was associated with less angiographic vasospasm and delayed ischemic neurological deficit than surgical clipping; furthermore, Dumont et al. still suggested whether this is attributable to differences in baseline risk factors between clipped and coiled patients or a true difference remained to be proven [21]. However, our study did not show better outcomes and less angiographic vasospasm for coiled patients. Thus, final conclusion remains to be verified.

Copeptin is co-synthesized with arginine vasopressin in the hypothalamus and is released into the portal circulation of the neurohypophysis. Arginine vasopressin contributes to the regulation of osmotic and cardiovascular homeostasis $[22,23]$. In addition, arginine vasopressin activates the hypothalamo - pituitary - adrenal axis through potentiation of corticotrophin - releasing - hormone induced adrenocorticotropic hormone secretion and thus reflects the individual stress response at a hypothalamic level $[24,25]$. Copeptin concentrations mirror that of arginine vasopressin [26]. Copeptin is known to have prognostic value in a variety of diseases, as it reflects disease severity and thus the chance of recovery. For example, copeptin levels have prognostic implications in patients with hemorrhagic and septic shock, lower respiratory tract infection and acute heart failure [1-11]. Therefore, it has been hypothesized that the close and reproducible relation of copeptin levels to the degree of activation of the stress axis is the basis of its unique usefulness as a prognostic biomarker [8]. In addition, data from experimental studies imply that vasopressin plays a role in brain edema formation as blocking of vasopressin receptors attenuates brain edema in ischemic and traumatic mice models [27-29]. The relationship between vasopressin levels and brain edema development has also been demonstrated in a clinical study of head injured patients [30]. Hence, the implication of copeptin and brain edema formation in $\mathrm{SAH}$ remains hypothetical at the moment.

Copeptin comes from the same precursor as mature arginine vasopressin, which is already well associated with hemodynamic changes and patient outcome. The measurement of mature arginine vasopressin, however, is subject to considerable challenges, and has therefore not reached clinical routine in the context of rapid measurements in the critically ill patients. Here, the stability and longer ex vivo half-life of copeptin is a practical advantage, which makes it easier to determine in the clinical laboratory. In this study, a receiver operating characteristic curve showed that plasma copeptin level on admission predicted poor functional outcome and mortality after 1 year, and in-hospital mortality of patients obviously. The area under curve of the copeptin concentration was similar to those of WFNS score and modified Fisher score for the prediction of these poor outcomes. In a combined 


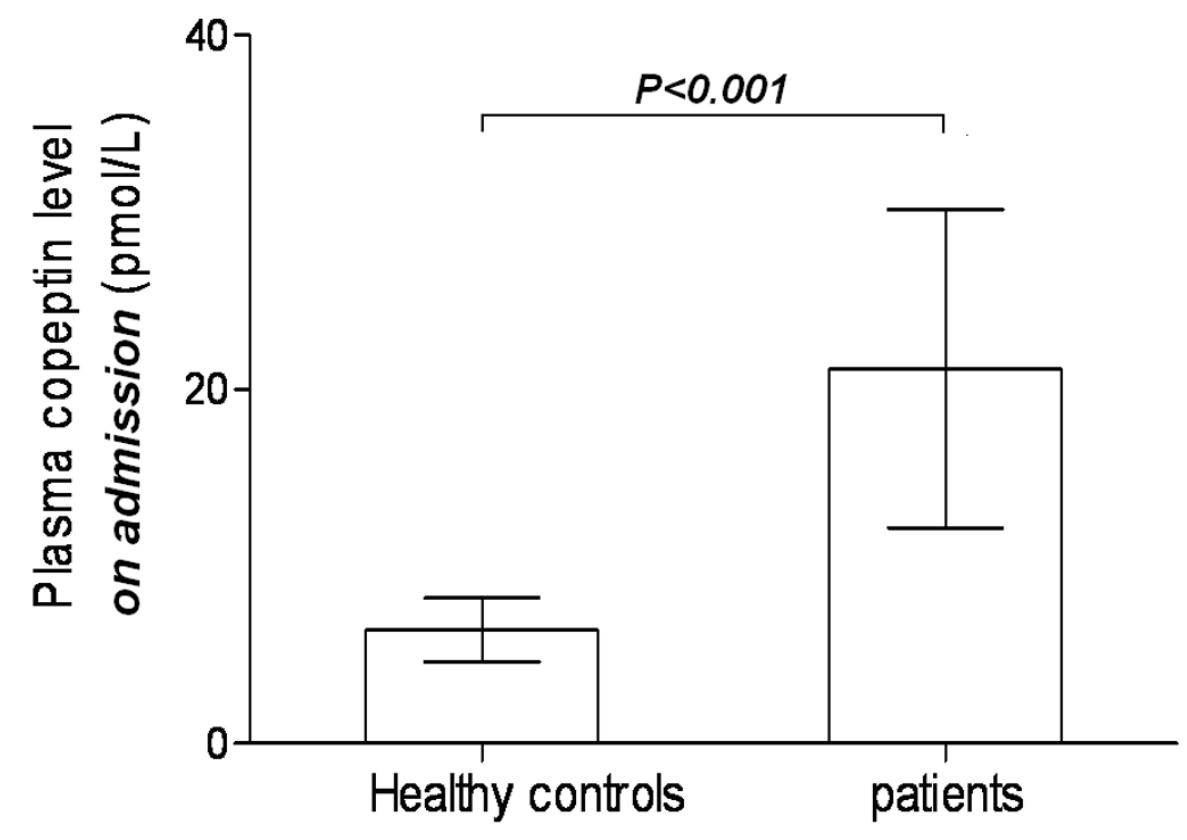

Figure 2 Graph showing the change of plasma copeptin concentration in the patients with aneurysmal subarachnoid hemorrhage. Data are expressed as mean \pm standard deviation.

Table 2 The factors associated with one-year mortality

\begin{tabular}{|c|c|c|c|}
\hline & $\begin{array}{c}\text { Non-survivals } \\
(n=42)\end{array}$ & $\begin{array}{c}\text { Survival } \\
(n=261)\end{array}$ & $P$ value \\
\hline Sex (male/female) & $18 / 24$ & $113 / 148$ & 0.958 \\
\hline Age (y) & $45.4 \pm 13.4$ & $43.6 \pm 12.2$ & 0.389 \\
\hline WFNS score on admission & $4.0 \pm 0.7$ & $2.1 \pm 1.0$ & $<0.001$ \\
\hline Modified Fisher score on admission & $4.2 \pm 0.6$ & $2.5 \pm 0.8$ & $<0.001$ \\
\hline Aneurysmal location & & & 0.614 \\
\hline Posterior communication artery & $8(19.0 \%)$ & 75 (28.7\%) & \\
\hline Internal carotid artery & $6(14.3 \%)$ & $37(14.2 \%)$ & \\
\hline Anterior communication artery & $9(21.4 \%)$ & $57(21.8 \%)$ & \\
\hline Middle cerebral artery & $7(16.7 \%)$ & $38(14.6 \%)$ & \\
\hline Anterior cerebral artery & $6(14.3 \%)$ & $29(11.1 \%)$ & \\
\hline Posterior cerebral artery & $4(9.5 \%)$ & $19(7.3 \%)$ & \\
\hline Vertebral artery & $2(4.8 \%)$ & $6(2.3 \%)$ & \\
\hline Surgery & $21(50.0 \%)$ & $165(63.2 \%)$ & 0.102 \\
\hline Aneurysmal size $(\mathrm{mm})$ & $11.1 \pm 5.3$ & $6.6 \pm 4.5$ & $<0.001$ \\
\hline Rebleeding & $10(23.8 \%)$ & $6(2.3 \%)$ & $<0.001$ \\
\hline Acute hydrocephalus & $25(59.5 \%)$ & $65(24.9 \%)$ & 0.001 \\
\hline Intracerebral hemorrhage & $19(45.2 \%)$ & $20(7.7 \%)$ & $<0.001$ \\
\hline Intraventricular hemorrhage & $37(88.1 \%)$ & $35(13.4 \%)$ & $<0.001$ \\
\hline External ventricular drain & $38(90.5 \%)$ & $71(27.2 \%)$ & $<0.001$ \\
\hline Angiographic vasospasm & $38(90.5 \%)$ & 93 (35.6\%) & $<0.001$ \\
\hline Computed tomography ischemia & $18(42.9 \%)$ & $32(12.3 \%)$ & $<0.001$ \\
\hline Admission time (hr) & $5.5 \pm 4.4$ & $4.6 \pm 3.5$ & 0.128 \\
\hline Seizure & $9(21.4 \%)$ & $35(13.4 \%)$ & 0.171 \\
\hline Plasma C-reactive protein level (mg/L) & $8.7 \pm 3.3$ & $6.9 \pm 2.6$ & $<0.001$ \\
\hline plasma D-dimer level (mg/L) & $2.4 \pm 1.0$ & $2.0 \pm 1.0$ & 0.015 \\
\hline Plasma copeptin level (pmol/L) & $31.4 \pm 8.3$ & $19.5 \pm 8.0$ & $<0.001$ \\
\hline
\end{tabular}

Numerical variables were presented as mean \pm standard deviation. Categorical variables were expressed as counts (percentage). Numerical variables were analyzed by Mann-Whitney U-test or unpaired Student $t$ test. Categorical variables were analyzed by chi-square test or Fisher exact test. $\mathrm{n}$ indicates number of patients; WFNS, World Federation of Neurological Surgeons. 
Table 3 Multivariate analysis of factors predicting the one-year mortality among 303 patients

\begin{tabular}{lccc}
\hline & Odds ratio & 95\% confidence interval & P value \\
\hline WFNS score on admission & 7.530 & $1.351 \sim 20.642$ & 0.002 \\
Modified Fisher score on admission & 9.181 & $2.236 \sim 22.297$ & 0.006 \\
Aneurysmal size (mm) & 1.182 & $0.914 \sim 1.324$ & 0.361 \\
Rebleeding & 4.243 & $0.624 \sim 26.748$ & 0.311 \\
Acute hydrocephalus & 1.249 & $0.230 \sim 25.387$ & 0.401 \\
Intracerebral hemorrhage & 1.235 & $0.642 \sim 6.891$ & 0.183 \\
Intraventricular hemorrhage & 3.822 & $0.893 \sim 11.402$ & 0.064 \\
External ventricular drain & 1.105 & $0.611 \sim 4.008$ & 0.397 \\
Angiographic vasospasm & 2.164 & $0.912 \sim 6.217$ & 0.132 \\
Computed tomography ischemia & 1.472 & $0.881 \sim 5.438$ & 0.271 \\
Plasma C-reactive protein level (mg/L) & 1.104 & $0.861 \sim 1.945$ & 0.401 \\
plasma D-dimer level (mg/L) & 0.941 & $0.573 \sim 1.908$ & 0.781 \\
Plasma copeptin level (pmol/L) & 2.307 & $1.324 \sim 6.974$ & 0.004 \\
\hline
\end{tabular}

The relation of copeptin to the mortality was assessed in a logistic-regression model. WFNS indicates World Federation of Neurological Surgeons.

logistic-regression model, copeptin improved the area under curve of WFNS score and modified Fisher score for the prediction of poor functional outcome after 1 year, but not for the prediction of mortality after 1 year or in-hospital mortality. Therefore, the determination of copeptin in the plasma of patients on admission provides the ability to distinguish between patients with good and bad outcome.
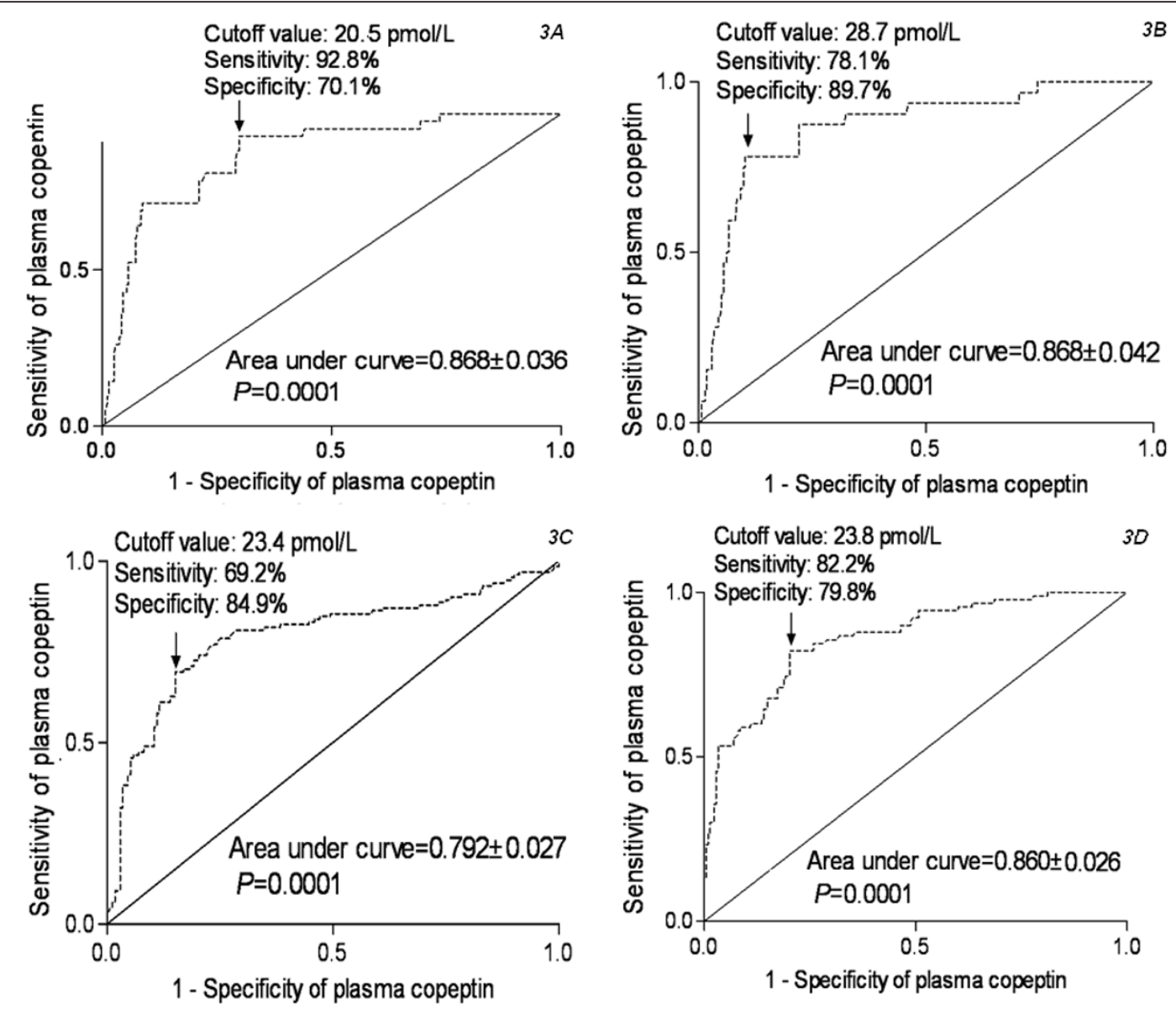

Figure 3 Graph showing receiver operating characteristic curve analysis of plasma copeptin level for one-year mortality (3A), inhospital mortality (3B), cerebrovasospasm (3C) and one-year poor functional outcome (3D). 
Table 4 Receiver operating characteristic curve analysis of factors predicting the one-year mortality among 303 patients

\begin{tabular}{lccc}
\hline & Copeptin & WFNS score & Modified Fisher score \\
\hline Criterion & $>20.5 \mathrm{pmol} / \mathrm{L}$ & $>3$ & $>3$ \\
Area under curve & 0.868 & 0.920 & 0.927 \\
95\% confidence interval & $0.824 \sim 0.904$ & $0.884 \sim 0.948$ & $0.892 \sim 0.954$ \\
Sensitivity & 92.9 & 78.6 & 88.1 \\
95\% confidence interval & $80.5 \sim 98.4$ & $63.2 \sim 89.7$ & $74.4 \sim 96.0$ \\
Specificity & 70.1 & 90.4 & 86.6 \\
$95 \%$ confidence interval & $64.2 \sim 75.6$ & $86.2 \sim 93.7$ & $81.8 \sim 90.5$ \\
Odds ratio & 29.949 & 34.613 & 47.743 \\
95\% confidence interval & $8.998 \sim 99.796$ & $14.876 \sim 80.563$ & $17.579 \sim 129.667$ \\
+ likelihood ratio & 3.11 & 8.20 & 6.57 \\
95\% confidence interval & $2.8 \sim 3.5$ & $7.0 \sim 9.7$ & $5.8 \sim 7.4$ \\
- likelihood ratio & 0.10 & 0.24 & 0.14 \\
95\% confidence interval & $0.03 \sim 0.3$ & $0.1 \sim 0.5$ & $0.06 \sim 0.3$ \\
$P$ value & Reference & 0.197 & 0.155 \\
\hline
\end{tabular}

WFNS indicates World Federation of Neurological Surgeons.

Table 5 The factors associated with in-hospital mortality

\begin{tabular}{|c|c|c|c|}
\hline & $\begin{array}{c}\text { Non-survivals } \\
(n=32)\end{array}$ & $\begin{array}{l}\text { Survival } \\
(n=271)\end{array}$ & $P$ value \\
\hline Sex (male/female) & $12 / 20$ & $119 / 152$ & 0.489 \\
\hline Age $(y)$ & $45.4 \pm 12.9$ & $43.7 \pm 12.3$ & 0.469 \\
\hline WFNS score on admission & $3.9 \pm 0.7$ & $2.2 \pm 1.1$ & $<0.001$ \\
\hline Modified Fisher score on admission & $4.3 \pm 0.6$ & $2.6 \pm 0.8$ & $<0.001$ \\
\hline Aneurysmal location & & & 0.599 \\
\hline Posterior communication artery & $5(15.6 \%)$ & $78(28.8 \%)$ & \\
\hline Internal carotid artery & $6(18.8 \%)$ & $37(13.7 \%)$ & \\
\hline Anterior communication artery & $8(25.0 \%)$ & $58(21.4 \%)$ & \\
\hline Middle cerebral artery & $4(12.5 \%)$ & $41(15.1 \%)$ & \\
\hline Anterior cerebral artery & $4(12.5 \%)$ & $31(11.4 \%)$ & \\
\hline Posterior cerebral artery & $3(9.4 \%)$ & $20(7.4 \%)$ & \\
\hline Vertebral artery & $2(6.3 \%)$ & $6(2.2 \%)$ & \\
\hline Surgery & $17(53.1 \%)$ & $169(62.4 \%)$ & 0.310 \\
\hline Aneurysmal size $(\mathrm{mm})$ & $11.9 \pm 5.1$ & $6.7 \pm 4.5$ & $<0.001$ \\
\hline Rebleeding & $6(18.8 \%)$ & $10(3.7 \%)$ & $<0.001$ \\
\hline Acute hydrocephalus & 19 (59.4\%) & $71(26.2 \%)$ & $<0.001$ \\
\hline Intracerebral hemorrhage & $15(46.9 \%)$ & $24(8.9 \%)$ & $<0.001$ \\
\hline Intraventricular hemorrhage & 31 (96.9\%) & $41(15.1 \%)$ & $<0.001$ \\
\hline External ventricular drain & $30(93.8 \%)$ & $79(29.2 \%)$ & $<0.001$ \\
\hline Angiographic vasospasm & $30(93.8 \%)$ & $101(37.3 \%)$ & $<0.001$ \\
\hline Computed tomography ischemia & $16(50.0 \%)$ & $34(12.6 \%)$ & $<0.001$ \\
\hline Admission time (hr) & $5.4 \pm 4.2$ & $4.6 \pm 3.6$ & 0.276 \\
\hline Seizure & 7 (21.9\%) & $37(13.7 \%)$ & 0.212 \\
\hline Plasma C-reactive protein level (mg/L) & $9.0 \pm 3.2$ & $6.9 \pm 2.6$ & $<0.001$ \\
\hline plasma D-dimer level (mg/L) & $2.4 \pm 1.1$ & $2.0 \pm 1.0$ & 0.045 \\
\hline Plasma copeptin level (pmol/L) & $31.8 \pm 8.5$ & $19.9 \pm 8.2$ & $<0.001$ \\
\hline
\end{tabular}

Numerical variables were presented as mean \pm standard deviation. Categorical variables were expressed as counts (percentage). Numerical variables were analyzed by Mann-Whitney U-test or unpaired Student $t$ test. Categorical variables were analyzed by chi-square test or Fisher exact test. $\mathrm{n}$ indicates number of patients; WFNS, World Federation of Neurological Surgeons. 
Table 6 Multivariate analysis of factors predicting the in-hospital mortality among 303 patients

\begin{tabular}{lccc}
\hline & Odds ratio & 95\% confidence interval & P value \\
\hline WFNS score on admission & 4.973 & $1.497 \sim 13.137$ & 0.003 \\
Modified Fisher score on admission & 5.982 & $2.469 \sim 17.149$ & 0.001 \\
Aneurysmal size (mm) & 1.218 & $0.909 \sim 1.423$ & 0.103 \\
Rebleeding & 1.483 & $0.882 \sim 5.492$ & 0.163 \\
Acute hydrocephalus & 1.097 & $0.711 \sim 4.483$ & 0.242 \\
Intracerebral hemorrhage & 1.479 & $0.703 \sim 5.176$ & 0.207 \\
Intraventricular hemorrhage & 3.94 & $0.816 \sim 9.407$ & 0.096 \\
External ventricular drain & 2.009 & $0.518 \sim 5.36$ & 0.148 \\
Angiographic vasospasm & 2.930 & $0.918 \sim 7.998$ & 0.091 \\
Computed tomography ischemia & 1.482 & $0.683 \sim 5.004$ & 0.315 \\
Plasma C-reactive protein level (mg/L) & 1.455 & $0.673 \sim 2.947$ & 0.482 \\
plasma D-dimer level (mg/L) & 0.915 & $0.587 \sim 2.936$ & 0.367 \\
Plasma copeptin level (pmol/L) & 2.515 & $1.399 \sim 8.229$ & 0.002 \\
\hline
\end{tabular}

The relation of copeptin to the mortality was assessed in a logistic-regression model. WFNS indicates World Federation of Neurological Surgeons.

Cerebrovasospasm is regarded as abnormal and prolonged smooth muscle contraction of cerebral arteries; many substances have been involved in the development of cerebral vasospasm following $\mathrm{SAH}$, but the complex mechanism of this arterial narrowing is not yet fully understood [31,32]. Some authors have reported that arginine vasopressin may play a role in the development of cerebral vasospasm [33,34] and ischemic brain edema [35,36]. Arginine vasopressin causes vasoconstriction in rabbit, feline, goat, rat and human [37-41]. In a model of SAH in rats, Delgado et al. demonstrated a biphasic, angiographically visible vasospasm with maximum acute vasospasm at $10 \mathrm{~min}$ after $\mathrm{SAH}$ and maximum late vasospasm 2 days later [42]. It was also shown that intracisternal injection of AVP determined acute vasospasm with a time-course similar to that seen in normal rats after SAH [34].
These studies sustain a better understanding of the role of arginine vasopressin in the cerebrovasospasm following SAH. Copeptin concentrations mirror that of arginine vasopressin [26]. Coupled with our observation that plasma copeptin level was an independent predictor for cerebrovasospasm of patients, the implication of copeptin and cerebrovasospasm formation in SAH remains hypothetical at the moment. Grade III is typically associated with the formation of vasospasm. This result was verified by receiver operating characteristic curve analysis with high area under curve. However, significantly lower accuracy for the prediction of cerebrovasospasm was found for plasma copeptin level compared with other clinical grade such as WFNS and modified Fisher grade. Hence, plasma levels of copeptin on admission are not recommended for the prediction of cerebrovasospasm after SAH.

Table 7 Receiver operating characteristic curve analysis of factors predicting the in-hospital mortality among 303 patients

\begin{tabular}{lccc}
\hline & Copeptin & WFNS score & Modified Fisher score \\
\hline Criterion & $>28.7$ pmol/L & $>3$ & $>3$ \\
Area under curve & 0.868 & 0.893 & 0.922 \\
$95 \%$ confidence interval & $0.825 \sim 0.904$ & $0.853 \sim 0.926$ & $0.886 \sim 0.950$ \\
Sensitivity & 78.1 & 75.0 & 90.6 \\
$95 \%$ confidence interval & $60.0 \sim 90.7$ & $56.6 \sim 88.5$ & $75.0 \sim 97.9$ \\
Specificity & 89.7 & 87.5 & 84.1 \\
$95 \%$ confidence interval & $85.4 \sim 93.0$ & $82.9 \sim 91.2$ & $79.2 \sim 88.3$ \\
Odds ratio & 29.798 & 20.911 & 51.254 \\
$95 \%$ confidence interval & $11.847 \sim 74.946$ & $8.698 \sim 50.270$ & $14.944 \sim 105.792$ \\
+ likelihood ratio & 7.56 & 5.98 & 5.71 \\
$95 \%$ confidence interval & $6.3 \sim 9.1$ & $4.9 \sim 7.3$ & $5.1 \sim 6.5$ \\
- likelihood ratio & 0.24 & 0.29 & 0.11 \\
95\% confidence interval & $0.1 \sim 0.5$ & $0.1 \sim 0.6$ & $0.04 \sim 0.3$ \\
$P$ value & Reference & 0.613 & 0.246 \\
\hline
\end{tabular}

WFNS indicates World Federation of Neurological Surgeons. 
Table 8 The factors associated with cerebrovasospasm

\begin{tabular}{|c|c|c|c|}
\hline & $\begin{array}{c}\text { Vasospasm } \\
(n=131)\end{array}$ & $\begin{array}{c}\text { Non-vasospasm } \\
(n=172)\end{array}$ & $P$ value \\
\hline Sex (male/female) & $61 / 70$ & $70 / 102$ & 0.307 \\
\hline Age (y) & $43.8 \pm 12.2$ & $43.9 \pm 12.5$ & 0.973 \\
\hline WFNS score on admission & $3.2 \pm 1.1$ & $1.7 \pm 0.9$ & $<0.001$ \\
\hline Modified Fisher score on admission & $3.5 \pm 1.0$ & $2.2 \pm 0.6$ & $<0.001$ \\
\hline Aneurysmal location & & & 0.813 \\
\hline Posterior communication artery & $33(25.2 \%)$ & $50(29.1 \%)$ & \\
\hline Internal carotid artery & $20(15.3 \%)$ & 23(13.4\%) & \\
\hline Anterior communication artery & $29(22.1 \%)$ & $37(21.5 \%)$ & \\
\hline Middle cerebral artery & $22(16.8 \%)$ & $23(13.4 \%)$ & \\
\hline Anterior cerebral artery & $16(12.2 \%)$ & $19(11.0 \%)$ & \\
\hline Posterior cerebral artery & $7(5.3 \%)$ & $16(9.3 \%)$ & \\
\hline Vertebral artery & $4(3.1 \%)$ & $4(2.3 \%)$ & \\
\hline Surgery & $86(65.5 \%)$ & $100(58.1 \%)$ & 0.183 \\
\hline Aneurysmal size $(\mathrm{mm})$ & $9.1 \pm 5.7$ & $5.8 \pm 3.5$ & $<0.001$ \\
\hline Rebleeding & $10(7.6 \%)$ & $6(3.5 \%)$ & 0.110 \\
\hline Acute hydrocephalus & $71(54.2 \%)$ & $19(11.0 \%)$ & $<0.001$ \\
\hline Intracerebral hemorrhage & $26(19.9 \%)$ & $13(7.6 \%)$ & 0.002 \\
\hline Intraventricular hemorrhage & $52(39.7 \%)$ & $20(11.6 \%)$ & $<0.001$ \\
\hline External ventricular drain & $90(68.7 \%)$ & $19(11.0 \%)$ & $<0.001$ \\
\hline Admission time (hr) & $4.5 \pm 3.4$ & $4.9 \pm 3.8$ & 0.342 \\
\hline Seizure & $17(13.0 \%)$ & $27(15.7 \%)$ & 0.505 \\
\hline Systolic arterial pressure $(\mathrm{mmHg})$ & $134.2 \pm 23.6$ & $128.7 \pm 21.2$ & 0.033 \\
\hline Diastolic arterial pressure $(\mathrm{mmHg})$ & $81.1 \pm 15.1$ & $77.5 \pm 13.8$ & 0.031 \\
\hline Mean arterial pressure $(\mathrm{mmHg})$ & $98.8 \pm 16.1$ & $94.6 \pm 15.3$ & 0.020 \\
\hline Plasma C-reactive protein level (mg/L) & $7.9 \pm 3.0$ & $6.6 \pm 2.4$ & $<0.001$ \\
\hline plasma D-dimer level (mg/L) & $2.2 \pm 1.1$ & $1.9 \pm 0.9$ & 0.011 \\
\hline Plasma copeptin level (pmol/L) & $25.0 \pm 8.4$ & $18.3 \pm 8.3$ & $<0.001$ \\
\hline
\end{tabular}

Numerical variables were presented as mean \pm standard deviation. Categorical variables were expressed as counts (percentage). Numerical variables were analyzed by Mann-Whitney U-test or unpaired Student $t$ test. Categorical variables were analyzed by chi-square test or Fisher exact test. $\mathrm{n}$ indicates number of patients; WFNS, World Federation of Neurological Surgeons.

Table 9 Multivariate analysis of factors predicting the cerebrovasospasm among 303 patients

\begin{tabular}{lccc}
\hline & Odds ratio & 95\% confidence interval & P value \\
\hline WFNS score on admission & 3.988 & $1.248 \sim 8.909$ & 0.005 \\
Modified Fisher score on admission & 4.692 & $1.627 \sim 15.726$ & 0.001 \\
Aneurysmal size (mm) & 1.104 & $0.998 \sim 1.247$ & 0.061 \\
Acute hydrocephalus & 1.548 & $0.932 \sim 4.877$ & 0.078 \\
Intracerebral hemorrhage & 1.709 & $0.437 \sim 5.174$ & 0.514 \\
Intraventricular hemorrhage & 2.472 & $0.911 \sim 6.972$ & 0.060 \\
External ventricular drain & 1.615 & $0.604 \sim 6.183$ & 0.176 \\
Systolic arterial pressure $(\mathrm{mmHg})$ & 1.012 & $0.989 \sim 1.214$ & 0.612 \\
Diastolic arterial pressure $(\mathrm{mmHg})$ & 1.003 & $0.972 \sim 1.044$ & 0.622 \\
Mean arterial pressure $(\mathrm{mmHg})$ & 1.005 & $0.901 \sim 1.103$ & 0.583 \\
Plasma C-reactive protein level $(\mathrm{mg} / \mathrm{L})$ & $0.713 \sim 1.082$ & 0.204 \\
plasma D-dimer level $(\mathrm{mg} / \mathrm{L})$ & 0.893 & $0.519 \sim 1.327$ & 0.595 \\
Plasma copeptin level $(\mathrm{pmol} / \mathrm{L})$ & 0.829 & $1.149 \sim 1.605$ & 0.025 \\
\hline
\end{tabular}

The relation of copeptin to the mortality was assessed in a logistic-regression model. WFNS indicates World Federation of Neurological Surgeons. 
Table 10 Receiver operating characteristic curve analysis of factors predicting the cerebrovasospasm among 303 patients

\begin{tabular}{lccc}
\hline & Copeptin & WFNS score & Modified Fisher score \\
\hline Criterion & $>23.4$ pmol/L & $>2$ & $>2$ \\
Area under curve & 0.792 & 0.879 & 0.874 \\
95\% confidence interval & $0.742 \sim 0.836$ & $0.837 \sim 0.913$ & $0.831 \sim 0.909$ \\
Sensitivity & 69.2 & 80.9 & 84.7 \\
95\% confidence interval & $60.8 \sim 77.4$ & $73.1 \sim 87.3$ & $77.4 \sim 90.4$ \\
Specificity & 84.9 & 79.7 & 76.7 \\
95\% confidence interval & $78.6 \sim 89.9$ & $72.9 \sim 85.4$ & $69.4 \sim 82.2$ \\
Odds ratio & 7.783 & 9.488 & 7.814 \\
95\% confidence interval & $4.617 \sim 13.121$ & $5.597 \sim 16.085$ & $4.663 \sim 13.096$ \\
+ likelihood ratio & 4.60 & 3.98 & 3.64 \\
95\% confidence interval & $4.0 \sim 5.2$ & $3.6 \sim 4.4$ & $3.3 \sim 4.1$ \\
- likelihood ratio & 0.36 & 0.24 & 0.20 \\
95\% confidence interval & $0.2 \sim 0.6$ & $0.2 \sim 0.4$ & $0.1 \sim 0.3$ \\
P value & Reference & 0.002 & 0.006 \\
\hline
\end{tabular}

WFNS indicates World Federation of Neurological Surgeons.

Table 11 The factors associated with one-year function outcome

\begin{tabular}{|c|c|c|c|}
\hline & $\begin{array}{l}\text { GOS } 1-3 \\
(n=90)\end{array}$ & $\begin{array}{c}\text { GOS 4-5 } \\
(n=213)\end{array}$ & $P$ value \\
\hline Sex (male/female) & $42 / 48$ & $89 / 124$ & 0.433 \\
\hline Age (y) & $44.7 \pm 11.3$ & $43.5 \pm 12.8$ & 0.422 \\
\hline WFNS score on admission & $3.6 \pm 0.7$ & $1.8 \pm 0.9$ & $<0.001$ \\
\hline Modified Fisher score on admission & $3.8 \pm 0.8$ & $2.3 \pm 0.7$ & $<0.001$ \\
\hline Aneurysmal location & & & 0.291 \\
\hline Posterior communication artery & $24(26.7 \%)$ & $59(27.7 \%)$ & \\
\hline Internal carotid artery & $13(14.4 \%)$ & $30(14.1 \%)$ & \\
\hline Anterior communication artery & 17 (18.9\%) & $49(23.0 \%)$ & \\
\hline Middle cerebral artery & $14(15.6 \%)$ & $31(14.6 \%)$ & \\
\hline Anterior cerebral artery & $7(7.8 \%)$ & $28(13.1 \%)$ & \\
\hline Posterior cerebral artery & $11(12.2 \%)$ & $12(5.6 \%)$ & \\
\hline Vertebral artery & $4(4.4 \%)$ & $4(1.9 \%)$ & \\
\hline Surgery & $54(60.0 \%)$ & $132(62.0 \%)$ & 0.747 \\
\hline Aneurysmal size $(\mathrm{mm})$ & $10.4 \pm 5.8$ & $5.9 \pm 3.7$ & $<0.001$ \\
\hline Rebleeding & $10(11.1 \%)$ & $6(2.8 \%)$ & 0.003 \\
\hline Acute hydrocephalus & $47(52.2 \%)$ & $43(20.2 \%)$ & $<0.001$ \\
\hline Intracerebral hemorrhage & $20(22.2 \%)$ & $19(8.9 \%)$ & 0.002 \\
\hline Intraventricular hemorrhage & $59(65.6 \%)$ & $12(5.6 \%)$ & $<0.001$ \\
\hline External ventricular drain & $66(73.3 \%)$ & $43(20.2 \%)$ & $<0.001$ \\
\hline Angiographic vasospasm & $71(78.9 \%)$ & $60(28.2 \%)$ & $<0.001$ \\
\hline Computed tomography ischemia & $28(31.1 \%)$ & $22(10.3 \%)$ & $<0.001$ \\
\hline Admission time (hr) & $4.5 \pm 3.5$ & $4.8 \pm 3.7$ & 0.577 \\
\hline Seizure & 19 (21.1\%) & $25(11.7 \%)$ & 0.085 \\
\hline Plasma C-reactive protein level (mg/L) & $8.2 \pm 3.2$ & $6.7 \pm 2.4$ & $<0.001$ \\
\hline plasma D-dimer level (mg/L) & $2.4 \pm 1.2$ & $1.9 \pm 0.9$ & $<0.001$ \\
\hline Plasma copeptin level (pmol/L) & $29.3 \pm 9.0$ & $17.8 \pm 6.4$ & $<0.001$ \\
\hline
\end{tabular}

Numerical variables were presented as mean \pm standard deviation. Categorical variables were expressed as counts (percentage). Numerical variables were analyzed by Mann-Whitney U-test or unpaired Student $t$ test. Categorical variables were analyzed by chi-square test or Fisher exact test. $\mathrm{n}$ indicates number of patients; WFNS, World Federation of Neurological Surgeons; GOS, Glasgow Outcome Scale. 
Table 12 Multivariate analysis of factors predicting one-year poor functional outcome among 303 patients

\begin{tabular}{lccc}
\hline & Odds ratio & 95\% confidence interval & P value \\
\hline WFNS score on admission & 4.930 & $1.997 \sim 13.438$ & 0.005 \\
Modified Fisher score on admission & 5.743 & $2.502 \sim 16.307$ & 0.001 \\
Aneurysmal size (mm) & 1.129 & $0.782 \sim 1.337$ & 0.137 \\
Rebleeding & 1.219 & $0.841 \sim 9.090$ & 0.103 \\
Acute hydrocephalus & 1.132 & $0.730 \sim 8.004$ & 0.218 \\
Intracerebral hemorrhage & 2.670 & $0.470 \sim 14.003$ & 0.314 \\
Intraventricular hemorrhage & 2.404 & $0.817 \sim 12.337$ & 0.087 \\
External ventricular drain & 1.316 & $0.718 \sim 4.796$ & 0.316 \\
Angiographic vasospasm & 1.174 & $0.790 \sim 3.931$ & 0.113 \\
Computed tomography ischemia & 1.422 & $0.849 \sim 3.640$ & 0.139 \\
Plasma C-reactive protein level (mg/L) & 1.019 & $0.827 \sim 1.213$ & 0.709 \\
plasma D-dimer level (mg/L) & 1.182 & $0.762 \sim 1.899$ & 0.418 \\
Plasma copeptin level (pmol/L) & 1.253 & $1.109 \sim 1.504$ & 0.001 \\
\hline
\end{tabular}

The relation of copeptin to the poor functional outcome was assessed in a logistic-regression model. WFNS indicates World Federation of Neurological Surgeons.

Table 13 Receiver operating characteristic curve analysis of factors predicting one-year poor functional outcome among 303 patients

\begin{tabular}{|c|c|c|c|}
\hline & Copeptin & WFNS score & Modified Fisher score \\
\hline Criterion & $>23.8 \mathrm{pmol} / \mathrm{L}$ & $>2$ & $>2$ \\
\hline Area under curve & 0.860 & 0.909 & 0.902 \\
\hline 95\% confidence interval & $0.815 \sim 0.897$ & $0.871 \sim 0.939$ & $0.863 \sim 0.933$ \\
\hline Sensitivity & 82.2 & 96.7 & 95.6 \\
\hline 95\% confidence interval & $72.7 \sim 89.5$ & $90.6 \sim 99.3$ & $89.0 \sim 98.7$ \\
\hline Specificity & 79.8 & 74.7 & 69.5 \\
\hline 95\% confidence interval & $73.8 \sim 85.0$ & $68.3 \sim 80.3$ & $62.8 \sim 75.6$ \\
\hline Odds ratio & 18.284 & 85.228 & 48.941 \\
\hline 95\% confidence interval & $9.684 \sim 34.522$ & $25.912 \sim 280.325$ & $17.237 \sim 139.015$ \\
\hline + likelihood ratio & 4.07 & 3.81 & 3.13 \\
\hline 95\% confidence interval & $3.6 \sim 4.6$ & $3.5 \sim 4.2$ & $2.8 \sim 3.5$ \\
\hline - likelihood ratio & 0.22 & 0.045 & 0.064 \\
\hline 95\% confidence interval & $0.1 \sim 0.4$ & $0.01 \sim 0.1$ & $0.02 \sim 0.2$ \\
\hline$P$ value & Reference & 0.109 & 0.178 \\
\hline
\end{tabular}

WFNS indicates World Federation of Neurological Surgeons.

\section{Conclusions}

In this study, plasma copeptin level is a useful, complementary tool to predict functional outcome and mortality after aneurysmal subarachnoid hemorrhage.

\section{Key messages}

- In the patients with aneurysmal subarachnoid hemorrhage, plasma copeptin level was substantially higher than that in healthy controls.

- Plasma copeptin level was an independent predictor of functional outcome and death after aneurysmal subarachnoid hemorrhage

- Copeptin level was a useful, complementary tool to predict functional outcome and mortality after aneurysmal subarachnoid hemorrhage.

\section{Abbreviations}

AUC: areas under the receiver operating characteristics curve; $C T$ : computerized tomography; ELISA: enzyme-linked immunosorbent assay; GOS: Glasgow outcome scale; SAH: subarachnoid hemorrhage; WFNS: World Federation of Neurological Surgeons.

\section{Authors' contributions}

XDZ and JSC contributed to the design of the study and drafted the manuscript and participated in the laboratory work. JSC, FZ and QCL enrolled the patients. GC and JMZ contributed to data analysis and interpretation of the results. All authors read and approved the final manuscript.

\section{Competing interests}

The authors declare that they have no competing interests.

Received: 22 August 2011 Accepted: 29 November 2011 Published: 29 November 2011 


\section{References}

1. Morgenthaler NG: Copeptin: a biomarker of cardiovascular and renal function. Congest Heart Fail 2010, 16:S37-44.

2. Morgenthaler NG, Müller B, Struck J, Bergmann A, Redl H, Christ-Crain M: Copeptin, a stable peptide of the arginine vasopressin precursor, is elevated in hemorrhagic and septic shock. Shock 2007, 28:219-226.

3. Müller B, Morgenthaler N, Stolz D, Schuetz P, Müller C, Bingisser R, Bergmann A, Tamm M, Christ-Crain M: Circulating levels of copeptin, a novel biomarker, in lower respiratory tract infections. Eur J Clin Invest 2007, 37:145-152.

4. Seligman R, Papassotiriou J, Morgenthaler NG, Meisner M, Teixeira PJZ: Copeptin, a novel prognostic biomarker in ventilator-associated pneumonia. Crit Care 2008, 12:R11.

5. Kelly D, Squire IB, Khan SQ, Quinn P, Struck J, Morgenthaler NG, Davies JE, $\mathrm{Ng}$ LL: C-terminal provasopressin (copeptin) is associated with left ventricular dysfunction, remodeling, and clinical heart failure in survivors of myocardial infarction. J Card Fail 2008, 14:739-745.

6. Stoiser B, Mörtl D, Hülsmann M, Berger R, Struck J, Morgenthaler NG, Bergmann A, Pacher R: Copeptin, a fragment of the vasopressin precursor, as a novel predictor of outcome in heart failure. Eur J Clin Invest 2006, 36:771-778.

7. Voors AA, von Haehling S, Anker SD, Hillege HL, Struck J, Hartmann O, Bergmann A, Squire I, van Veldhuisen DJ, Dickstein K, OPTIMAAL Investigators: C-terminal provasopressin (copeptin) is a strong prognostic marker in patients with heart failure after an acute myocardial infarction: results from the OPTIMAAL study. Eur Heart J 2009, 30:1187-1194.

8. Katan M, Fluri F, Morgenthaler NG, Schuetz P, Zweifel C, Bingisser R, Müller K, Meckel S, Gass A, Kappos L, Steck AJ, Engelter ST, Müller B, ChristCrain M: Copeptin: a novel, independent prognostic marker in patients with ischemic stroke. Ann Neurol 2009, 66:799-808.

9. Urwyler SA, Schuetz P, Fluri F, Morgenthaler NG, Zweifel C, Bergmann A, Bingisser R, Kappos Lb, Steck Ab, Engelter Sb, Müller Bf, Christ-Crain M, Katan M: Prognostic value of copeptin: one-year outcome in patients with acute stroke. Stroke 2010, 41:1564-1567.

10. Zweifel C, Katan M, Schuetz P, Siegemund M, Morgenthaler NG, Merlo A, Mueller B, Christ-Crain M: Copeptin is associated with mortality and outcome in patients with acute intracerebral hemorrhage. BMC Neurol 2010, 10:34.

11. Kleindienst A, Brabant G, Morgenthaler NG, Dixit KC, Parsch $H$, Buchfelder M: Following brain trauma, copeptin, a stable peptide derived from the AVP precusor, does not reflect osmoregulation but correlates with injury severity. Acta Neurochir Suppl 2010, 106:221-224.

12. Dong $X Q$, Huang $M$, Yang $S B$, Yu WH, Zhang $Z Y$ : Copeptin is associated with mortality in patients with traumatic brain injury. J Trauma 2011.

13. Dong XQ, Huang M, Yu WH, Zhang ZY, Zhu Q, Che ZH, Du Q, Wang H: Change in plasma copeptin level after acute spontaneous basal ganglia hemorrhage. Peptides 2011, 32:253-257.

14. Drake C: Report of World Federation of Neurological Surgeons Committee on a universal subarachnoid hemorrhage grading scale. $J$ Neurosurg 1988, 68:985-986.

15. Fisher CM, Kistler JP, Davis JM: Relation of cerebral vasospasm to subarachnoid hemorrhage visualized by computerized tomographic scanning. Neurosurgery 1980, 6:1-9.

16. Jennett $B$, Bond $M$ : Assessment of outcome after severe brain damage. Lancet 1975, 1:480-484

17. Katan M, Müller B, Christ-Crain M: Copeptin: a new and promising diagnostic and prognostic marker. Crit Care 2008, 12:117.

18. Rosen DS, Macdonald RL: Grading of subarachnoid hemorrhage: modification of the world World Federation of Neurosurgical Societies scale on the basis of data for a large series of patients. Neurosurgery 2004, 54:566-575.

19. Juvela S, Siironen J: D-dimer as an independent predictor for poor outcome after aneurysmal subarachnoid hemorrhage. Stroke 2006, 37:1451-1456

20. Fountas KN, Tasiou A, Kapsalaki EZ, Paterakis KN, Grigorian AA, Lee GP Robinson JS Jr: Serum and cerebrospinal fluid C-reactive protein levels as predictors of vasospasm in aneurysmal subarachnoid hemorrhage. Neurosurg Focus 2009, 26:E22

21. Dumont AS, Crowley RW, Monteith SJ, llodigwe D, Kassell NF, Mayer S, Ruefenacht D, Weidauer S, Pasqualin A, Macdonald RL: Endovascular treatment or neurosurgical clipping of ruptured intracranial aneurysms: effect on angiographic vasospasm, delayed ischemic neurological deficit, cerebral infarction, and clinical outcome. Stroke 2010, 41:2519-2524.

22. Itoi K, Jiang YQ, Iwasaki Y, Watson SJ: Regulatory mechanisms of corticotrophin - releasing hormone and vasopressin gene expression in the hypothalamus. J Neuroendocrinol 2004, 16:348-355.

23. Asfar $P$, Hauser B, Radermacher $P$, Matejovic M: Catecholamines and vasopressin during critical illness. Crit Care Clin 2006, 22:131-149.

24. Itoi K, Seasholtz AF, Watson SJ: Cellular and extracellular regulatory mechanisms of hypothalamic corticotropin-releasing hormone neurons. Endocr J 1998, 45:13-33.

25. Katan M, Morgenthaler N, Widmer I, Puder JJ, Konig C, Muller B, ChristCrain M: Copeptin, a stable peptide derived from the vasopressin precursor, correlates with the individual stress level. Neuro Endocrinol Lett 2008, 29:341-346.

26. Struck J, Morgenthaler NG, Bergmann A: Copeptin, a stable peptide derived from the vasopressin precursor, is elevated in serum of sepsis patients. Peptides 2005, 26:2500-2504.

27. Vakili A, Kataoka H, Plesnila N: Role of arginine vasopressin V1 and V2 receptors for brain damage after transient focal cerebral ischemia. J Cereb Blood Flow Metab 2005, 25:1012-1019.

28. Molnar AH, Varga C, Berko A, Rojik I, Parducz A, Laszlo F, Laszlo FA: Inhibitory effect of vasopressin receptor antagonist OPC-31260 on experimental brain oedema induced by global cerebral ischaemia. Acta neurochirurgica 2008, 150:265-271

29. Trabold R, Krieg S, Scholler K, Plesnila N: Role of vasopressin V(1a) and V2 receptors for the development of secondary brain damage after traumatic brain injury in mice. Journal of neurotrauma 2008, 25:1459-1465.

30. Xu M, Su W, Huang WD, Lu YQ, Xu QP, Chen ZJ: Effect of AVP on brain edema following traumatic brain injury. Chinese journal of traumatology 2007, 10:90-93.

31. Sobey CG, Faraci FM: Subarachnoid haemorrhage: What happens to the cerebral arteries? Clin Exp Pharmacol Physiol 1998, 25:867-876.

32. Cook DA: Mechanisms of cerebral vasospasm in subarachnoid haemorrhage. Pharmacol Ther 1995, 66:259-284.

33. Cach R, Durboraw C, Smock T, Albeck D: Microvascular spasm is mediated by vasopressin fibers in the rat hippocampal slice. Brain Res 1989 483:221-225

34. Delgado TJ, Abdul-Rahman Arbab M, Warberg J, Svendgaard NA: The role of vasopressin in acute cerebral vasospasm. J Neurosurg 1988, 68:266-273.

35. Doczi T, Laszlo FA, Szerdahelyi P, Joo P: Involvement of vasopressin in brain edema formation: Further evidence obtained from the Brattleboro diabetes insipidus rat with experimental subarachnoid hemorrhage. Neurosurgery 1984, 14:436-441.

36. Shuaib A, Wang CX, Yang T, Noor R: Effects of nonpeptide V1 vasopressin receptor antagonist SR-49059 on infarction volume and recovery of function in a focal embolic stroke model. Stroke 2002, 33:3033-3037.

37. Fernández N, Martínez MA, García-Villalón AL, Monge L, Diéguez G: Cerebral vasoconstriction produced by vasopressin in conscious goats. Role of vasopressin V1 and V2 receptors and nitric oxide. Br J Pharmacol 2001, 132:1837-1844

38. Garcia-Villalon AL, Garcia JL, Fernandez N, Monge L, Gomez B, Dieguez G: Regional differences in the arterial response to vasopressin: Role of endothelial nitric oxide. Br J Pharmacol 1996, 118:1848-1854.

39. Katori E, Ohta T, Nakazato $Y$, Ito S: Vasopressin-induced contraction in the basilar artery in vitro. Eur J Pharmacol 2001, 416:113-121.

40. Kurahashi K, Wang A, Koshino Y, Nishihashi T: Arginine vasopressininduced contraction of rat basilar artery: Involvement of endothelium, reactive oxygen species, 5-lipoxygenase and leukotriene B4. Jpn J Pharmacol 2002, 88:267.

41. Tsuji T, Cook DA: Vasoconstrictor mechanism of neuropeptides augmented after endothelial removal in isolated, perfused canine basilar arteries. Neurol Res 1995, 17:193-200.

42. Delgado TJ, Brismar J, Svendgaard NA: Subarachnoid haemorrhage in the rat: Angiography and fluorescence microscopy of the major cerebral arteries. Stroke 1985, 16:595-602.

doi:10.1186/cc10575

Cite this article as: Zhu et al:: Detection of copeptin in peripheral blood of patients with aneurysmal subarachnoid hemorrhage. Critical Care 2011 15:R288. 